\title{
Multimodal Therapy for Painful Bladder Syndrome / Interstitial Cystitis: Pilot Study Combining Behavioral, Pharmacologic, and Endoscopic Therapies
}

\author{
Robert S. Hanley, John T. Stoffel, Ralph M. Zagha, Arthur Mourtzinos, John F. Bresette
}

Anne Arundel Urology (RSH), Annapolis, Maryland, Department of Urology (JTS, AM, JFB), Lahey Clinic Medical Center, Burlington, Massachusetts, Department of Urology (RMZ), Florida Medical Center, Lauderdale Lakes, Florida, USA

\begin{abstract}
Purpose: We evaluated the effectiveness of combining behavioral therapy, pharmacologic therapy and endoscopic hydrodistension for treating painful bladder syndrome / interstitial cystitis (PBS/IC).

Materials and Methods: Twenty-five patients with PBS/IC were prospectively enrolled in a pilot multimodal behavioral, pharmacologic and endoscopic treatment protocol. Behavioral modification included diet recommendations, fluid restriction to $64 \mathrm{oz}$. /day, progressive timed voiding and Kegel exercises. Oral pharmacologic therapy consisted of daily doses of macrodantin $100 \mathrm{mg}$, hydroxyzine 10-20 mg and urised 4 tablets. Patients underwent endoscopic bladder hydrodistention under anesthesia at least 2 weeks after protocol enrollment. Behavioral and pharmacological treatments were continued after the hydrodistention. O'Leary-Sant questionnaire scores were recorded before starting the protocol, after pharmacologic/behavioral therapy, 2 months post-hydrodistension, and at scheduled follow-up.

Results: Eighteen patients (72\%) completed the pilot multimodal treatment protocol and were followed for a mean of 10.2 months. All patients were female with a median age of 36.3 years and had mean bladder capacity under anesthesia of 836 milliliters. Mean O'Leary-Sant symptom index scores for baseline symptoms, after behavioral/pharmacologic treatment, post-hydrodistension and during follow up were 12.5, 8.6, 7.0, and 6.7 ( $\mathrm{p}<0.05$ ). Mean O'Leary-Sant problem index scores for baseline, after behavioral/pharmacologic treatment, post-hydrodistention and during follow up were 12.7, 8.9, 6.7 , and $7.7(\mathrm{p}<0.05)$.

Conclusion: Our pilot multimodal protocol of behavioral modification, pharmacologic therapy and endoscopic hydrodistention demonstrated a significant progressive improvement in PBS/IC quality of life scores, compared to a pre-treatment baseline. These results should be validated in a larger, placebo controlled trial.
\end{abstract}

Key words: cystitis, interstitial; pilot project; behavioral medicine; endoscopy; pharmacology

Int Braz J Urol. 2009; 35: 467-74

\section{INTRODUCTION}

Painful bladder syndrome / interstitial cystitis (PBS/IC) is defined by the International Continence Society as "suprapubic pain related to bladder filling, accompanied by other symptoms such as increased day- and nighttime frequency, in the absence of proven urinary infection or other obvious pathology. Interstitial cystitis is a PBS characterized by cystoscopic and morphological findings not further defined." (1). The pathophysiology of PBS/IC remains unclear and investigators have attributed the severe symptoms to 
a history of chronic urinary tract infections, leaky glycosaminoglycan layers in the bladder, autoimmune inflammation, and/or neurogenic inflammation (2). Because of the uncertainty surrounding the underlying PBS/IC pathophysiology, there are multiple treatment options for patients with severe symptoms. In general, PBS/IC treatments have focused on behavioral modifications, pharmacotherapy, or endoscopic treatments. However, the efficacy of each type of treatment is highly variable when examined as monotherapy (3).

In fact, $\mathrm{PBS} / \mathrm{IC}$ may represent several different etiologies presenting with common symptomatic endpoints. Consequently, multimodal, treatment for PBS/IC may improve overall efficacy because different pathophysiologies may require different treatment modalities. Currently, there is little information in the literature on a multimodal approach for treating PBS/IC. We have developed a pilot treatment program that offers a simple combination of common, easy to implement behavioral modification, pharmacologic, and endoscopic therapies for PBS/IC. The goal of this pilot study was to determine if this combination of multimodality therapy offered consistent, measurable relief for female patients presenting with previously untreated symptomatic PBS/IC.

\section{MATERIALS AND METHODS}

Female patients referred to the urogynecology clinics of three physicians for irritative or painful bladder symptoms were screened for PBS/IC using the ICS PBS/IC definition. The IC Database Study requirements were also utilized for exclusion criteria to rule out confounding pathologies (4), Table-1. Prior to pilot study enrollment, all patients underwent a complete history and physical examination to rule out other potential sources for bladder symptoms and all patients submitted urine specimens for culture and cytology. Only female patients with a new diagnosis of $\mathrm{PBS} / \mathrm{IC}$ and the absence of other urologic pathologies were included in the study. Patients unable to complete questionnaires or unwilling to agree to a scheduled treatment plan were excluded from the study. The study was approved by the institutional IRB.

The multimodal therapy used in this study consisted of three established PBS/IC treatments: behavioral modification, pharmacologic therapy, and endoscopic hydrodistension. Behavioral modification and pharmacologic therapy were started at the time of patient enrollment. Endoscopic therapy was initiated a minimum of 2 weeks afterwards.

\section{Behavioral Modification}

PBS/IC patients enrolled in the study were given 30 minutes of specific verbal instruction by an urogynecology physician or nurse using a set script. Patients were instructed to perform progressive timed voiding on a 2 to 3 hour schedule and instructed to limit liquid intake to $64 \mathrm{oz}$. per day, divided into 16 oz. per meal and 8 oz. between meals. For patients unable to hold urine for this interval, instructions were given to progressively increase urine storage time between voids by 15 minutes per week until the goal of a 2 to 3 hour interval was reached. All patients were also instructed to perform 15 pelvic muscle Kegal contractions twice a day. At the conclusion of the teaching session, all patients were given

Table 1 - Exclusion criteria from IC database study entry requirements.

\section{Exclusion Criteria}

1. No history of current genitor-urinary tuberculosis

2. No history of urethral cancer

3. No history of bladder malignancy, high grade dysplasia, or carcinoma in situ

4. Females:

a. No occurrence of ovarian, vaginal, or cervical cancer in the past three years

b. No current vaginitis, clue cell, trichomonas, or yeast infection

5. No bacterial cystitis in the previous three months

6. No active herpes in the previous three months

7. No antibiotics for culture proven urinary tract infection in previous three months

8. No previous treatment with cyclophosphamide (cytoxan)

9. No radiation cystitis

10. No neurogenic bladder dysfunction

11. No bladder outlet obstruction 
written instructions that summarized these behavioral regimens. An exclusionary list of foods that may exacerbate PBS/IC symptoms was discussed (5). If a patient identified a specific food or foods from the list that they believed may increase her bladder symptoms, recommendations were given to avoid this substance. At each successive clinic visit, an urogynecology nurse questioned the patient regarding compliance to each element of the behavioral therapy regimen and deviation from recommended therapy was noted.

\section{Pharmacologic Therapy}

At the first visit, all patients were also instructed on a specific oral pharmacologic regimen that included macrodantin $100 \mathrm{mg}$ daily, hydroxyzine 10 to $20 \mathrm{mg}$ daily, and Urised (methenamine, methylene blue, phenyl salicylate, benzoic acid, atropine sulfate, hyoscyamine) 4 tablets daily. Patients also continued pentosan polysulfate sodium $100 \mathrm{mg}$ three times per day if their primary care physician had started this medication 6 months prior to the first urogynecology visit. Medication compliance was assessed by a urogynecology nurse at each clinical follow-up. Medication side effects and deviation from recommended therapy was recorded. No patients were prescribed additional narcotics/analgesics during the study.

\section{Endoscopic Hydrodistention}

At a minimum 2 weeks after initiating behavioral/pharmacologic therapy, each patient was evaluated via a standardized cystoscopic protocol. After a successful general anesthesia, a $21 \mathrm{~F}$ cystoscope was inserted per urethra and the bladder was surveyed. The bladder was then filled to capacity with sterile water via gravity irrigation $(100 \mathrm{~cm} \mathrm{H} 20$ above pubic symphysis). The bladder was then emptied and the bladder surveyed again with the cystoscope. Mucosal glomerulization and Hunner's ulcers were specifically recorded, if present. Hydrodistension was repeated three times at bladder capacity. Bladder capacity under anesthesia for the three distensions was averaged and recorded as maximum bladder capacity.

\section{Outcome Assessment}

The severity of PBS/IC symptoms before and after interventions was assessed using the validated O'Leary-Sant Interstitial Cystitis questionnaire. This robust questionnaire is an 8 item form divided into two domains assessing symptom severity (Symptom Index - 4 questions) and the impact of interstitial cystitis (IC) on daily life (Problem Index - 4 questions). Each question is scored by the patient with higher numbers in each domain representing greater severity and impact. Maximum Symptom and Problem Index scores were 20 and 16, respectively (6). The patients were given the O'Leary-Sant questionnaire at initial clinical presentation. The questionnaires were again administered after 1 month of behavioral and pharmacologic therapy treatment. Patients returned within 2 months after hydrodistension for questionnaire completion and were scheduled for return visits every three to six months afterwards for questionnaire completion. Voiding diaries were not utilized.

Paired t-tests and ANOVA were used to analyze the data. Statistical significance was defined as $p$ $<0.05$. All tests were performed with statistical software SPSS v13.0 (SPSS, Inc., Chicago, IL, USA).

\section{RESULTS}

Twenty-five patients were prospectively enrolled in the study between July 2004 and August 2006. Patients had PBS/IC symptoms for a median 12 months (range 3-60) prior to presentation at our institution. Eighteen (72\%) completed the pilot multimodal treatment protocol and returned questionnaires for evaluation. Of the 7 patients who were excluded prior to analysis, 3 patients did not wish to continue the behavioral therapy and did not wish to pursue hydrodistension. Two patients were lost to follow-up prior to hydrodistension, 1 patient was excluded due to newly diagnosed pelvic endometriosis during the study and 1 patient was excluded after transitional cell carcinoma of the bladder was found during the endoscopic hydrodistension.

The median age at presentation for these patients was 36.3 years (SD 15.9) and the most common presenting symptom was urinary urgency (18 
patients), followed by urinary frequency (17 patients) and pelvic pain (16 patients). Associated comorbidities, based on diagnosis from qualified treating physicians, included depression $(67 \%)$, irritable bowel syndrome (28\%), anxiety (28\%), inflammatory bowel disease (17\%), and fibromyalgia (17\%).

All patients completing the protocol reported good compliance with the behavioral fluid management/timed voiding/Kegel exercise regimen and there were no marked protocol deviations. Seventeen patients remained on Urised (94\%), and 16 on hydroxizine and macrodantin (89\%) throughout the study. Patients withdrawing from the suggested medications cited gastrointestinal irritation (urised - 1 patient, macrodantin - 1 patient) and dizziness (hydroxyzine - 1 patient) as precipitating factors. Three patients reported Elmiron usage for greater than 6 months prior to initiation of treatment and were maintained on the prescribed medication.

All 18 patients underwent hydrodistention under anesthesia at a mean of 2.1 months (SD 1.3) from the initiation of behavioral and pharmacologic therapy. Significant glomerulations during cystoscopy were seen in 17 patients (94\%). No Hunner's ulcers were identified. The mean maximum bladder capacity under anesthesia was 836 milliliters (SD 154). All patients tolerated the hydrodistension and were restarted on their exact behavioral and pharmacologic regimens post-operatively. There were no adverse events reported from the hydrodistension.

Mean follow-up for patients completing the protocol was 10.2 months (CI 95\% [5.7, 14.7]). The mean initial symptom and problem index scores prior to initiating the combined protocol were 12.5 (CI $95 \%[10.9,14.1])$ and 12.7 (CI [11.4, 14.1]). After behavioral modification and pharmacologic therapy but prior to hydrodistension, 18 patients completed questionnaires. Mean symptom and problem index scores were 8.5 ([6.8, 10.2]) and 8.9 ([7.2, 10.7]). At 2 months after hydrodistension, 14 patients completed questionnaires and mean symptom and problem index scores were $7.0([5.0,9.0])$ and $6.7([5.0,8.5])$, respectively. At last follow-up after hydrodistension, 7 patients had available data. Mean symptom and problem indexes for these patients were 6.7 ([4.7, $8.8]$ ) and 7.7 ([4.9, 10.5]). Changes in symptom (p $<0.001)$ and problem index scores $(\mathrm{p}<0.001)$ from baseline were significant. Data are summarized in Figure-1. The greatest change in problem and symptom scores was seen in the interval between baseline and of behavioral/pharmacologic therapy (Figure-2).

\section{COMMENTS}

In this study, we prospectively evaluated symptomatic PBS/IC patients treated with a unique pilot multimodal program, consisting of behavioral, pharmacologic, and endoscopic hydrodistension therapy. We demonstrated significant statistical improvement in QOL scores over baseline after initiating the behavioral/pharmacologic therapy, maintenance of this improvement after hydrodistension and sustainable relief for months afterwards. This is the first study, to our knowledge, to demonstrate efficacy for a prospectively evaluated multimodal behavioral, pharmacologic, endoscopic protocol. Based on these initial results, a larger randomized trial investigating the efficacy of the individual protocol components appears justified.

Literature on combined protocols for treating $\mathrm{PBS} / \mathrm{IC}$ is generally limited (7-9). Consequently, we can not compare our outcomes to other multimodal therapies.

However, physicians commonly use multiple modalities for treating PBS/IC. In Rovner et al.'s review of the Interstitial Cystitis Data Base (ICDB), these authors found over 180 different types of PBS/ IC treatments, with $21 \%, 34 \%$, and $28 \%$ of women treated via single-mode, a combination of two, and three or more different types of therapies, respectively (10). Consequently, our pilot study suggests more research should be performed to prospectively investigate the efficacy of multimodal approaches.

The results of our multimodal therapy compare favorably with single modality therapies. Pentosanpolysulfate (PPS) therapy, the oral heparin analogue, showed a $26 \%$ subjective improvement in a placebo controlled multi-center trial (11). Amitriptyline therapy has a similar efficacy in the literature. In one of the largest prospective, randomized, placebo-controlled, double-blind study, the mean total symptom score decreased from 26.9 to 18.5 (31\%) in the amitriptyline group compared with 27.6 - 24.1 


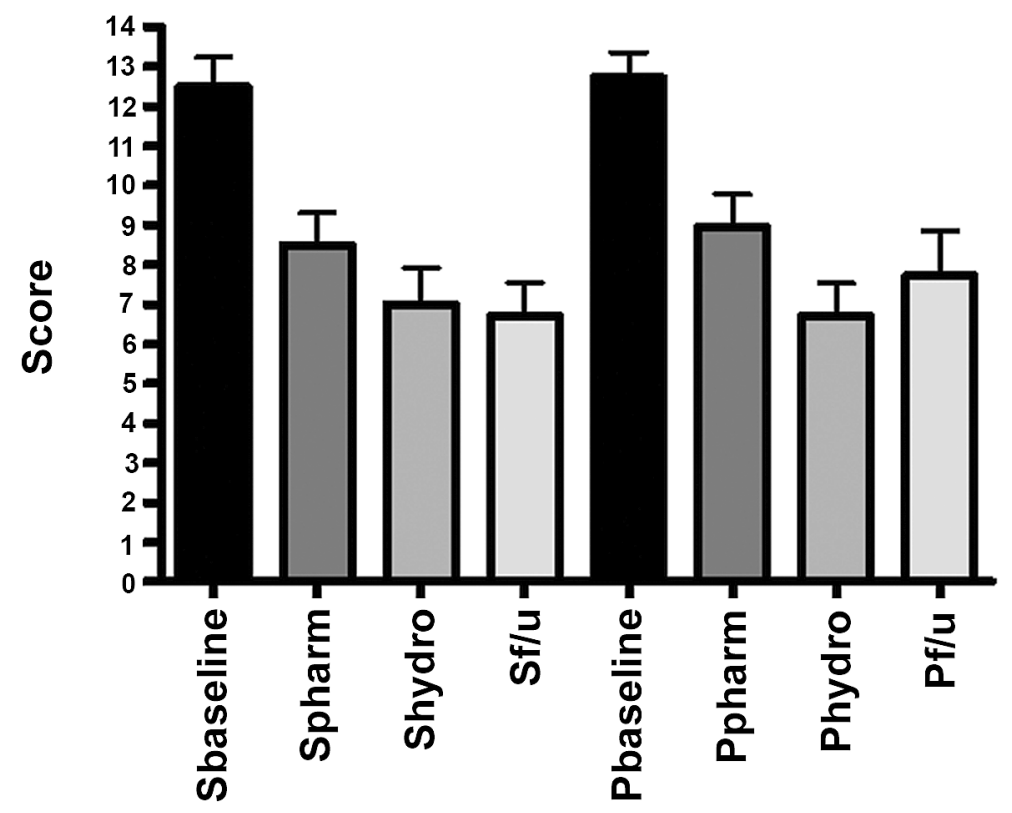

Figure 1 - Mean O'Leary-Sant Symptom and Problem Index Scores at each time in combined protocol. Sbaseline, Pbaseline = prior to any treatment; Spharm, Ppharm = after behavioral/pharmacologic treatment; Shydro, Phydro = after hydrodistention; $S f / u, P f / u=$ scores at last follow-up after hydrodistention.

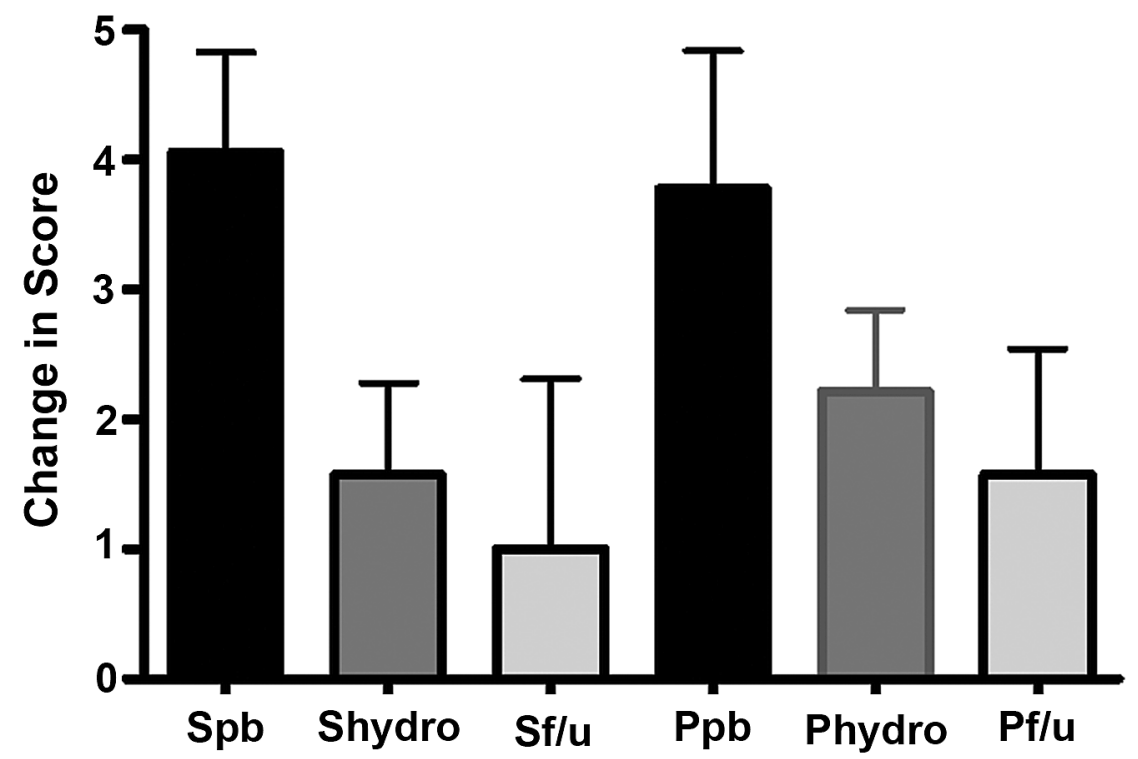

Figure 2 - Change in O'Leary-Sant Symptom and Problem Index Scores compared to previous visit.

$S p b, P p b=$ change in symptom, problem score after pharmacologic and behavioral therapy compared to baseline; Shydro, Phydro = change in symptom, problem score after hydrodistention, compared to score after pharmacologic/behavioral therapy; Sf/u,Pf/u = change in symptom, problem score at last follow up, compared to score after hydrodistention. 
in the placebo group, $p=0.005,(12)$. Initial results from our multimodality protocol also appear at least as effective as intravesical therapy, another mainstay therapy for PBS/IC (13). Since neither PPS, amitriptyline, nor intravesical therapy has gained universal use among practitioners, our protocol, if validated, may provide further treatment options for frustrated physicians and patients.

We selected modalities for our protocol based on ease of implementation and historical reports of efficacy. At the first visit, we begin with behavioral modification that focuses on timed voiding and fluid management because there is some evidence that these strategies may play some role in symptom reduction. Chaikan et al. had 42 patients with refractory symptoms undergo fluid management/timed voiding and showed an decrease in urinary frequency from 17 voids per day to 8 voids per day (14). Although true evidence based evaluations of these behavioral measures are lacking in the literature, Whitmore suggests that behavioral changes may, at minimum, empower the patient and improve coping mechanisms (15). We noted almost universal compliance and self-reported improvement with timed voiding, fluid restriction, food exclusion and pelvic floor exercises and strongly concur with this suggestion.

We prescribe three medications, hydroxyzine, macrobid, and urised (methenamine, methylene blue, phenyl salicylate, benzoic acid, atropine sulfate, hyoscyamine) as part of our multimodality plan. We selected hydroxyzine based on the theory that it is a mast cell stabilizer and thus may play an important role in mediating the inflammatory process observed in PBS/IC (16). As an adjunct to hydroxyzine, we also utilized macrodantin. Although not commonly considered first line therapies for PBS/IC, some studies suggest that some cases of PBS/IC may be due to dormant microbes or an infectious etiology (17). Finally, we utilize Urised as an antispasmodic. Little information is available on antispasmodic use for treating PBS/IC, although Hill recently reported that patients taking phenothiazide consistently reported PBS/IC symptom reduction while on this medication (18). This observation matches our own clinical experience.

As the final treatment arm of our multimodal therapy plan, we performed endoscopic hydrodisten- sion under anesthesia a mean 2.1 months after starting the behavioral and pharmacologic treatments. Although somewhat controversial, some studies suggest that endoscopic hydrodistention has (short term) efficacy in the treatment of PBS/IC symptoms. Ottem $\&$ Teichman, retrospectively reviewed 84 consecutive PBS/IC patients treated with hydrodistension and found over $50 \%$ reported symptomatic improvement 2 months after the procedure (19). Unfortunately, however, it is difficult to generalize results from hydrodistension studies due to variance in techniques and outcome reporting. In our study, patients showed significant, if small, improvement in quality of life after undergoing hydrodistension. Consequently, we believe adding this procedure to the regimen of behavioral and pharmacologic therapy yields benefit for the symptomatic PBS/IC patient.

As with any observational study examining $\mathrm{PBS} / \mathrm{IC}$ treatments, this study has limitations. We recognize that the PBS/IC subjects in our study may not be representative of the general $\mathrm{PBS} / \mathrm{IC}$ population since the general population is difficult to standardize. For example, we utilized the PBS/IC Study Data Base recommendations as inclusion and exclusion criteria and the mean bladder capacity in our study was 836 $\mathrm{mL}$. In contrast, studies using the NIDDK inclusion/ exclusion criteria for diagnosing PBS/IC would have excluded all patients with a bladder capacity greater than $350 \mathrm{~mL}$. Furthermore, none of our patients had mucosal ulcerations on cystoscopic examination. In other studies, the prevalence of mucosal ulceration ranges as high as $20 \%(20)$.

Outcomes in our study were measured via change in the validated O'Leary-Sant IC questionnaire. We chose this outcome measure because voiding diaries hold too much internal variability to be reliable measures for a small efficacy study. Currently, there are three published PBS/IC quality of life questionnaires: the O'Leary-Sant IC Symptom Index and IC Problem Index, the University Of Wisconsin IC Scale, and the Pelvic Pain and Urgency/ Frequency Scale. In theory, investigators employing different questionnaires may yield interpretations of outcomes.

Although data from this study is limited to 18 patients, the initial data from this pilot study is encouraging. Significant statistical improvement 
in PBS/IC quality of life scores was noted for these patients both after starting behavior/pharmacologic and after hydrodistension. Despite this clinically significant improvement, we recognize that these observations could be confounded by a potential placebo effect and by spontaneous remission of PBS/IC symptoms. In general, placebo controlled trials are lacking in PBS/IC research and the placebo effect can be difficult to quantify even in randomized controlled PBS/IC trials. Spontaneous remission rates certainly should be considered when interpreting outcomes from any PBS/IC study, even though the epidemiology of spontaneous remission is poorly understood or studied. Since there is no solid research documenting remission rates or timeframes, we can not speculate on how our data was effected other than comment that outcomes appear durable in our limited data. Further investigation with blinded, randomized trials is needed to better understand any potential placebo effect.

\section{CONCLUSION}

This pilot trial suggests that symptomatic PBS/ IC patients treated with a multimodal treatment plan consisting of behavioral, pharmacologic, and endoscopic therapy demonstrate significant, progressive, and durable improvement. A placebo controlled trial with longer follow up is needed to validate these findings.

\section{ACKNOWLEDGEMENTS}

Robert S. Hanley and John T. Stoffel contributed equally to the manuscript and share first authorship.

\section{CONFLICT OF INTEREST}

None declared.

\section{REFERENCES}

1. Abrams P, Cardozo L, Fall M, Griffiths D, Rosier P, Ulmsten U, et al.: The standardisation of terminol- ogy of lower urinary tract function: report from the Standardisation Sub-committee of the International Continence Society. Neurourol Urodyn. 2002; 21: 167-78.

2. Koziol JA, Clark DC, Gittes RF, Tan EM: The natural history of interstitial cystitis: a survey of 374 patients. J Urol. 1993; 149: 465-9.

3. Kelada E, Jones A: Interstitial cystitis. Arch Gynecol Obstet. 2007; 275: 223-9.

4. Simon LJ, Landis JR, Tomaszewski JE, Nyberg LM: The Interstitial Cstitis Database (ICDB) Study. In: Interstitial Cystitis, Sant GR (ed.). Philadelphia, Lippincott-Raven. 1997; pp. 17-24.

5. Gillespie L: Interstitial Cystitis and Diet. In: Interstitial Cystitis, Sant GR (ed.). Philadelphia, Lippincott-Raven. 1997, pp. 111.

6. O'Leary MP, Sant GR, Fowler FJ Jr, Whitmore KE, Spolarich-Kroll J: The interstitial cystitis symptom index and problem index. Urology. 1997; 49(5A Suppl): 58-63.

7. Liu HT, Kuo HC: Intravesical botulinum toxin A injections plus hydrodistension can reduce nerve growth factor production and control bladder pain in interstitial cystitis. Urology. 2007; 70: 463-8.

8. Baykal K, Senkul T, Sen B, Karademir K, Adayener C, Erden D: Intravesical heparin and peripheral neuromodulation on interstitial cystitis. Urol Int. 2005; 74: 361-4.

9. Dell JR, Butrick CW: Multimodal therapy for painful bladder syndrome/interstitial cystitis. J Reprod Med. 2006; 51(3 Suppl): 253-60.

10. Rovner E, Propert KJ, Brensinger C, Wein AJ, Foy M, Kirkemo A, et al.: Treatments used in women with interstitial cystitis: the interstitial cystitis data base (ICDB) study experience. The Interstitial Cystitis Data Base Study Group. Urology. 2000; 56: 940-5.

11. Mulholland SG, Hanno P, Parsons CL, Sant GR, Staskin DR: Pentosan polysulfate sodium for therapy of interstitial cystitis. A double-blind placebo-controlled clinical study. Urology. 1990; 35: 552-8.

12. van Ophoven A, Pokupic S, Heinecke A, Hertle L: A prospective, randomized, placebo controlled, doubleblind study of amitriptyline for the treatment of interstitial cystitis. J Urol. 2004; 172: 533-6.

13. Dawson TE, Jamison J: Intravesical treatments for painful bladder syndrome/ interstitial cystitis. Cochrane Database Syst Rev. 2007; 17: CD006113.

14. Chaiken DC, Blaivas JG, Blaivas ST: Behavioral therapy for the treatment of refractory interstitial cystitis. J Urol. 1993; 149: 1445-8. 


\section{Multimodal Therapy for Interstitial Cystitis: A Pilot Study}

15. Whitmore KE: Self-care regimens for patients with interstitial cystitis. Urol Clin North Am. 1994; 21: 121-30.

16. Theoharides TC, Sant GR: Hydroxyzine therapy for interstitial cystitis. Urology. 1997; 49(5A Suppl): 10810.

17. Domingue GJ, Ghoniem GM, Bost KL, Fermin C, Human LG: Dormant microbes in interstitial cystitis. J Urol. 1995; 153: 1321-6. Erratum in: J Urol 1996; 155: 298.
18. Hill JR, Isom-Batz G, Panagopoulos G, Zakariasen K, Kavaler E: Patient perceived outcomes of treatments used for interstitial cystitis. Urology. 2008; 71: 62-6.

19. Ottem DP, Teichman JM: What is the value of cystoscopy with hydrodistension for interstitial cystitis? Urology. 2005; 66: 494-9.

20. Koziol JA, Adams HP, Frutos A: Discrimination between the ulcerous and the nonulcerous forms of interstitial cystitis by noninvasive findings. J Urol. 1996; 155: 87-90.

Accepted after revision:

March 6, 2009

\section{Correspondence address:}

Dr. John T. Stoffel

Departmentof Urology

Lahey Clinic

41 Mall Road

Burlington, MA, 01805, USA

E-mail: john.t.stoffel@lahey.org 